\section{Hipopituitarismo Associado a Hipoglicemia: Relato de 2 Casos e Revisão da Literatura}

A ocorrência de hipoglicemia associada a hipopituitarismo é evento raro em pacientes adultos e um pouco mais freqüente em crianças portadoras de disfunção pituitária semelhante. Nos pacientes portadores de insuficiência adrenal, a hipoglicemia é mais prevalente naqueles cuja deficiência de cortisol é secundária à deficiência de ACTH do que naqueles com doença adrenal primária. Isto se deve, provavelmente, ao fato de que, na primeira condição, existem deficiências simultâneas de $\mathrm{GH}$ e cortisol. Entretanto, hipoglicemia pode ocorrer nos casos com deficiência isolada de ACTH. Vamos apresentar, inicialmente, dois casos de pacientes adultos, do sexo masculino, com hipopituitarismo e hipoglicemia. A seguir, discutiremos os possiveis mecanismos envolvidos na hipoglicemia, a etiologia mais provável do hipopituitarismo e algumas particularidades presentes nos dois pacientes.

Descritores: Hipoglicemia; Hipopituitarismo; Insuficiência adrenal secundária; Hipofisite linfocitária

\section{APRESENTAÇÃO DOS CASOS}

\section{Caso 1}

$\mathbf{H}^{2}$ OMEM De 30 anOs, COM história de 2 anos de episódios de perda de consciência no período de jejum matinal, que melhoravam com a injeção endovenosa de glicose. Apresentava, concomitantemente, anorexia, perda de peso gradual $(4 \mathrm{~kg})$, fraqueza discreta e tontura ao assumir a posição ortostática. Durante um desses episódios foi atendido no HCFMUSP, com glicemia capilar de $17 \mathrm{mg} / \mathrm{dL}$ e em coma superficial, que reverteu após a injeção endovenosa de glicose. $\mathrm{O}$ exame físico era normal exceto por enrijecimento bilateral dos pavilhões auriculares. Não apresentava hipotensão postural e não havia nenhuma evidência de desidratação.

\section{Investigação Laboratorial}

Exames gerais: anemia normocítica-normocrômica discreta, eletrólitos normais e funções renal e hepática sem alterações. Durante jejum alimentar de 12 a 16 horas, o paciente apresentava hipoglicemias associadas a níveis baixos de insulina (tabela 1 ). A determinação de anticorpo antiinsulina foi negativa.

Avaliação endocrinológica: 1) ACTH: $<21 \mathrm{pg} / \mathrm{ml}(\mathrm{VN}: \leq 60 \mathrm{pg} / \mathrm{ml})$ e cortisol (8hs): $<\mathrm{l} \mu \mathrm{g} / \mathrm{dl}$ (VN: 7-3l $\mu \mathrm{g} / \mathrm{dL}) ; 2) \mathrm{GH}$ : 0,6ng/dl $(\mathrm{VN}$ : $<5 \mathrm{ng} / \mathrm{mL}$ ) e IGF-I: 27 e $57 \mathrm{ng} / \mathrm{dl}(\mathrm{VN}: 74-399 \mathrm{ng} / \mathrm{dl}) ; 3)$ TSH: $25 \mu \mathrm{U} / \mathrm{ml}$ (VN: $0,5-4,5 \mu \mathrm{U} / \mathrm{mL}$ ) e T4-livre: $0,5 \mathrm{ng} / \mathrm{dl}$ (VN: 0,6 a $1,5 \mathrm{ng} / \mathrm{dl}$ ); 4) LH: 6,4UI/L (VN: 1,4-9,2UI/L), FSH: 2,1UI/L (VN: 1-12UI/L), testosterona: 75lng/dl (VN: 240 a 1030ng/dL) e Prolactina: 10,9ng/dl (VN: $<14,5 \mathrm{ng} / \mathrm{dL}$ ); 5) DHEA: 1,2ng/dl (VN: 3-5,7ng/ml), DHEAS: $202 \mathrm{ng} / \mathrm{ml}$ (VN: 468-333lng/ml), androstenediona: $0,5 \mathrm{ng} / \mathrm{ml}$ (VN: $1,1-2 \mathrm{ng} / \mathrm{ml}$ ), aldosterona: $<2,5 \mathrm{ng} / \mathrm{dl}$ (VN/posição supina: $9-12 \mathrm{ng} / \mathrm{dl}$ ) e atividade de reni-

\section{caso especial}

\author{
Catarina B. D'Alva Rocha \\ Maria Adelaide A. Pereira
}

\author{
Serviço de Endocrinologia e \\ Metabologia, Hospital das \\ Clínicas da Faculdade de \\ Medicina da Universidade de \\ São Paulo (HC-FMUSP), \\ São Paulo, SP.
}

Recebido em 13/12/01

Revisado em 25/03/02 Aceito em 03/05/02 
na plasmática (APR): $<0,2 \mathrm{ng} / \mathrm{ml} / \mathrm{h}$ ( $\mathrm{VN} /$ posição supina: $0,2-2,8 \mathrm{ng} / \mathrm{ml} / \mathrm{h})$.

A administração EV de $250 \mathrm{mg}$ de Cortrosina simples não elevou o cortisol que permaneceu inferior a $\mathrm{lmg} / \mathrm{dl}$. Cortrosina depot $(500 \mu \mathrm{g} / 2 \mathrm{X} \mathrm{dia} / 2$ dias) aumentou o cortisol para $9,6 \mu \mathrm{g} / \mathrm{dl}$. A injeção de TRH $(200 \mu \mathrm{g}$ EV) aumentou maximamente o TSH aos $30 \mathrm{~m}(32 \mu \mathrm{UI} / \mathrm{ml})$ que permaneceu elevado até os $90 \mathrm{~m}$ após a injeção $(24 \mu \mathrm{UI} / \mathrm{ml})$. Os anticorpos antitireoideanos (antiTPO e anti tireoglobulina) foram negativos. A USG de tireóide revelou tireóide de contornos regulares, de textura heterogênea com peso total de $8,5 \mathrm{~g}$.

A ressonância magnética $(\mathrm{RM})$ revelou hipófise normal com espessamento discreto da haste (figura 1 ). A tomografia de abdome demonstrou adrenais de volume diminuído.

\section{Caso 2}

Homem de 57 anos, com história de 9 anos de episódios de perda da consciência, que melhoravam com a infusão de soro glicosado. Apresentava fraqueza progressiva, astenia, anorexia e perda de peso. Notou durante esse período endurecimento dos pavilhões auriculares. O restante do exame físico era normal. Não havia hipotensão postural.

\section{Avaliação Laboratorial}

Exames gerais: hemograma, eletrólitos e provas de funções hepática e renal sem anormalidades. Durante períodos de jejum alimentar de até lohs o paciente apresentava níveis baixos de glicose e insulina (tabela 1$)$.

\begin{tabular}{ccc}
\multicolumn{3}{l}{ Tabela 1. Glicemia e Insulina (casos 1 e 2). } \\
\hline $\begin{array}{c}\text { JEJUM } \\
\text { (hs) }\end{array}$ & $\begin{array}{c}\text { GLICEMIA } \\
(\mathrm{mg} / \mathrm{dl})\end{array}$ & $\begin{array}{c}\text { INSULINA } \\
(\mu \mathrm{UI} / \mathrm{ml})\end{array}$ \\
\hline $\begin{array}{c}\text { Caso } 1 \\
12\end{array}$ & 37 & \\
16 & 23 & $\mathrm{NR}$ \\
16 & 16 & $<5$ \\
Caso 2 & & $<5$ \\
10 & 68 & $<5$ \\
10 & 54 & $<5$ \\
\hline
\end{tabular}

Valores normais (jejum de 12hs): insulina: $<20 \mu \mathrm{Ul} / \mathrm{ML}$ NR: não realizado

Avaliação endocrinológica: 1) ACTH: $<24 \mathrm{pg} / \mathrm{ml}$ (VN: $\leq 60 \mathrm{pg} / \mathrm{ml}$ ) e cortisol: $<\mathrm{l} \mu \mathrm{g} / \mathrm{dl}$ (VN: 7-3l $\mu \mathrm{g} / \mathrm{dL}$ ); 2) GH: <lng/dl e IGF-I: $2 \operatorname{lng} / \mathrm{dl}$ (VN: 55-252ng/dl); 3) TSH: $18 \mu \mathrm{UI} / \mathrm{ml}(\mathrm{VN}: 0,5-4,5 \mu \mathrm{UI} / \mathrm{ml})$ e T4-livre: $0,5 \mathrm{ng} / \mathrm{dl}(\mathrm{VN}: 0,6-1,5 \mathrm{ng} / \mathrm{dL})$; 4) LH: 8,5UI/L (VN: 1,4-9,2UI/L), FSH: 6,8UI/L (VN: 1-12UI/L), testosterona: 759ng/dl (VN: 240-1030ng/dL) e Prolactina: $15 \mathrm{ng} / \mathrm{dl}$ (VN: $<14,5 \mathrm{ng} / \mathrm{dL}$ ); 5) DHEA: $0,7 \mathrm{ng} / \mathrm{mL}$ (VN: 3-5,7ng/mL), DHEAS: <100ng/ml (VN: $468-3331 \mathrm{ng} / \mathrm{ml}$ ) e androstenediona: $0,4 \mathrm{ng} / \mathrm{ml}$ (VN: 1,1-2ng/ml).

Não houve resposta do cortisol após a admi-

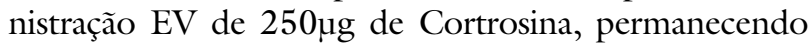

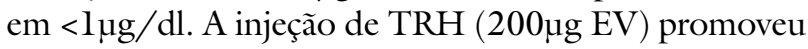
resposta positiva e prolongada do TSH ao TRH, alcançando valores de $43 \mu \mathrm{UI} / \mathrm{ml}$ aos $30 \mathrm{~m}$ e $27 \mu \mathrm{UI} / \mathrm{ml}$ aos $90 \mathrm{~m}$.Os anticorpos antitireoideanos foram negativos e a US revelou tireóide com redução de volume $(5,8 \mathrm{~g})$. A RM de hipófise revelou haste hipofisária com espessura no limite superior da normalidade.

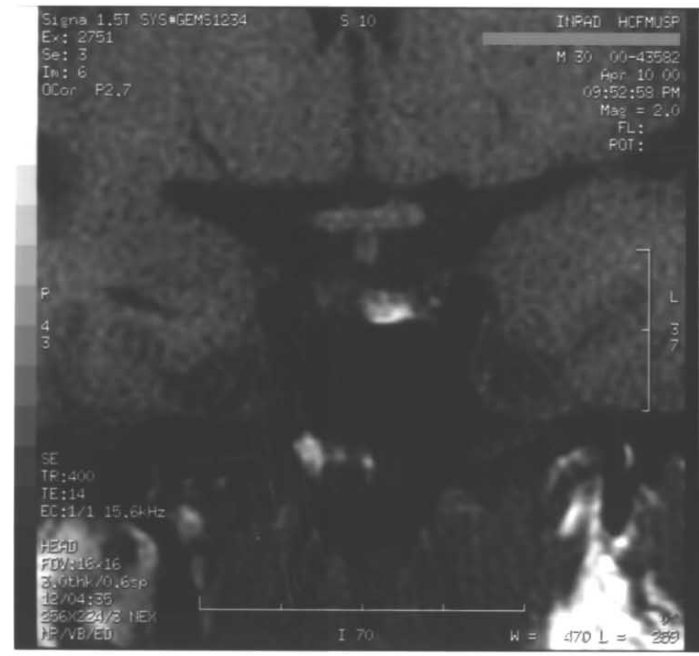

Fig. A

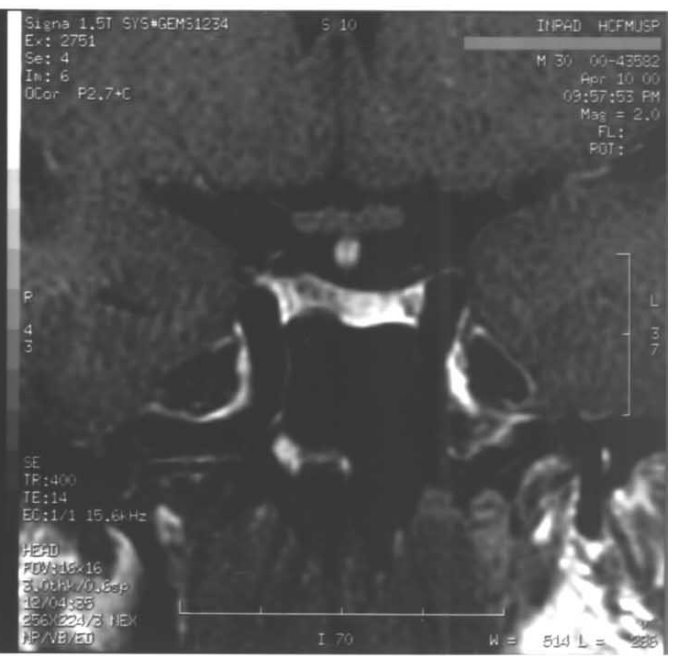

Fig. B

Figura 1. Ressonância magnética: hipófise de dimensões normais e haste discretamente espessada (A: T1 sem contraste e B: T1 com contraste). 


\section{DISCUSSÃO}

Apresentamos 2 casos de pacientes adultos, do sexo masculino, com hipoglicemia severa não dependente de insulina, associada a hipopituitarismo parcial, representado por deficiência grave do setor corticotrófico e deficiência de hormônio de crescimento(GH). Não havia alterações no setor gonadotrófico e lactotrofo. Os dois pacientes apresentavam T4 livre baixo com TSH elevado.

Algumas das causas mais freqüentes de hipoglicemia são: 1- Uso de drogas hipoglicemiantes. Esta eventualidade foi excluída nos nossos pacientes, pela anamnese; 2- Insulinoma ou Hipoglicemia Pancreatogênica não relacionada ao Insulinoma. Essas possibilidades foram afastadas pela presença de níveis baixos de insulina na vigência de hipoglicemia, 3Insuficiências Hepática, Renal e Cardíaca, excluídas pela investigação clínica e laboratorial; 4- Neoplasias produtoras de IGF-II. Embora não tenha sido possível realizar as dosagens de IGF-II, essa causa de hipoglicemia nos pareceu bastante improvável porque, além da falta de evidências dos tumores que, com maior freqüência, se associam à produção de IGF-II, os pacientes apresentavam outras alterações que, como veremos, podem justificar a hipoglicemia; 5- Hipoglicemia de origem autoimune. Essa possibilidade foi afastada, parcialmente, no primeiro paciente, com a dosagem de anticorpo anti-insulina; 6- Deficiência de hormônios contra-reguladores da ação da insulina. Esta, como discutiremos a seguir, é a causa mais provável da hipoglicemia apresentada por nossos pacientes.

A hipoglicemia causada pela deficiência de hormônios contra-reguladores não é uma situação clínica comum em pacientes adultos com hipopituitarismo, exceto quando, ao hipopituitarismo, se associa diabetes tratado com insulina ou hipolicemiantes orais. Nesses pacientes, a existência concomitante de resposta deficitária do glucagon e catecolaminas, que ocorre em alguns diabéticos, não permite recuperação de hipoglicemia induzida, em geral, pelo tratamento do diabetes (1).

Normalmente, a recuperação da queda glicêmica induzida por jejum ou medicação se deve a três mecanismos: 1- Supressão da secreção endógena de insulina. Este é o evento mais importante para a recuperação da glicemia e ocorre quando são atingidos níveis glicêmicos ao redor de 76 a $72 \mathrm{mg} / \mathrm{dl} ; 2$ - Liberação dos hormônios contra-reguladores, que estimulam a glicogenólise e neoglicogênese e, conseqüentemente, aumentam a produção endógena de glicose. A su- pressão da insulina induzida pela queda da glicemia também estimula esses mesmos processos; 3-Mobilização de substratos (ácidos graxos livres e aminoácidos) que são precursores da neoglicogênese. Isto ocorre pelo estímulo da proteólise e lipólise, provocado tanto pela queda da insulina como pela elevação de alguns hormônios contra-reguladores (catecolaminas). Além disso, o aumento de GH, cortisol e catecolaminas provoca resistência periférica à ação da insulina e o aumento de catecolaminas reduz, ainda mais, a secreção de insulina, já bloqueada pela queda glicêmica (1).

A resposta dos vários hormônios à hipoglicemia obedece a uma escala hierárquica na qual o primeiro evento é a supressão da secreção endógena da insulina e o segundo é a liberação dos hormônios contra-reguladores. Portanto, durante a queda da glicemia, a diminuição da insulina e a liberação dos hormônios contrareguladores promovem, pelos mecanismos expostos anteriormente, a recuperação dos níveis glicêmicos.

No modelo de hipoglicemia aguda, induzida pela administração em bolo de insulina endovenosa, verificamos que a recuperação da glicemia coincide com a liberação de glucagon, epinefrina, GH e de cortisol. Utilizando-se, isoladamente, bloqueadores da ação ou da liberação desses hormônios, com o objetivo de avaliar sua contribuição individual nesse processo, verifica-se que a deficiência de glucagon isolada ou de glucagon e catecolaminas são os estados que mais comprometem a recuperação da glicemia (1). Num segundo modelo de hipoglicemia prolongada induzida pela infusão endovenosa contínua de insulina $\left(15 \mathrm{mU} / \mathrm{m}^{2} / \mathrm{min} \mathrm{SC}\right)$ observa-se que não há recuperação da glicemia, apesar de haver produção mantida dos hormônios contra-reguladores (glucagon, catecolaminas, GH e cortisol). Quando estudamos a participação de cada hormônio contra-regulador verificamos o seguinte: num indivíduo controle a infusão contínua de insulina causa queda inicial da glicemia que, posteriormente, se estabiliza em níveis de $60 \mathrm{mg} / \mathrm{dL}$ às custas de aumento na produção endógena de glicose. $\mathrm{O}$ bloqueio da secreção de glucagon impede o aumento da produção endógena de glicose e leva à hipoglicemia severa $(40 \mathrm{mg} / \mathrm{dL})$. O aumento dos outros hormônios contrarreguladores não compensa o déficit de glucagon. Quando as secreções de GH e cortisol são bloqueadas também ocorre hipoglicemia grave, só que mais tardia, em torno de 4 horas após o início da infusão. Portanto, o glucagon e as catecolaminas, hormônios liberados precocemente, atuam imediatamente após a instalação da hipoglicemia, enquanto o GH e o cortisol, hormônios liberados posteriormente, atuam numa fase mais tardia da recuperação glicêmica (2). 
A partir desses experimentos podemos concluir que o evento mais importante na recuperação glicêmica é a dissipação da ação da insulina, embora a presença dos hormônios contra-reguladores torne essa recuperação mais rápida e eficaz. Quando não se permite a dissipação da insulina (por infusão mantida), podemos verificar com maior clareza o papel fundamental desses hormônios na normalização da glicemia.

$O$ fato de não observarmos hipoglicemia grave em pacientes adultos com hipopituitarismo talvez possa ser explicado porque o bloqueio da secreção de insulina é o principal fator envolvido na recuperação da hipoglicemia. Esses pacientes, embora tendam a apresentar glicemias mais baixas, não desenvolvem hipoglicemia severa após jejum de 12 horas porque ocorre supressão fisiológica da secreção de insulina. Entretanto, crianças com hipopituitarismo apresentam, com maior freqüência, hipoglicemia durante períodos de jejum e isto se deve, provavelmente, à menor eficácia do fígado na produção endógena de glicose.

Os dois pacientes apresentados têm deficiência de cortisol e GH e apresentam hipoglicemias severas relacionadas ao jejum. A pergunta que formulamos é a seguinte: por que esses pacientes desenvolveram hipoglicemia grave no período de jejum matinal se normalmente os pacientes adultos com hipopituitarismo não o fazem?

Existem poucos relatos de casos semelhantes na literatura. Nos últimos 20 anos encontramos descrições de 8 pacientes adultos com hipoglicemia associada a hipopituitarismo. Vamos, a seguir, descrever 3 desses pacientes, que tiveram um estudo mais completo. $\mathrm{O}$ primeiro deles é uma paciente do sexo feminino, de 31 anos, que iniciou, no período pós-parto, quadro de anorexia, perda de peso, fraqueza e dor abdominal. Teve um episódio de taquicardia e tremores seguido de rebaixamento do nível de consciência e nesta ocasião foi detectada glicemia de $40 \mathrm{mg} / \mathrm{dl}$. Houve recuperação da consciência após infusão de glicose e, alguns dias após, durante episódio semelhante ela evoluiu com agitação, cianose, parada cardíaca e óbito. Os achados da necrópsia dessa paciente foram de uma hipófise reduzida de tamanho com infiltração linfocítica no lobo anterior, adrenais com atrofia importante das camadas fascicular e reticular, tireóide infiltrada por células monucleares e ovários normais. Portanto, foram feitos os diagnósticos de hipofisite e tireoidite linfocitárias (3). O segundo caso é de uma paciente do sexo feminino, de 18 anos que, 18 meses após o parto, foi hospitalizada por rebaixamento do nível de consciência e glicemia de $22 \mathrm{mg} / \mathrm{dL}$. Desde o puerpério a paciente vinha apresentando anorexia, perda de peso, fraqueza e episódios intermitentes de confusão mental. A avaliação funcional da adenohipófise demonstrou perda de função tireotrófica, corticotrófica, somatotrófica e lactotrófica com preservação do setor gonadotrófico. A tomografia de sela túrcica foi normal e houve desaparecimento dos episódios hipoglicêmcios com a reposição de glicocorticóides. Portanto, tratava-se de hipopituitarismo parcial, com preservação da função gonadotrófica, que se desenvolveu no pós-parto (4). O terceiro caso é o de paciente do sexo feminino, de 23 anos que apresentou hipoglicemia grave com quadro de convulsão seguido de coma, na $28^{a}$ semana de gestação. Após esse primeiro evento apresentou outros episódios hipoglicêmicos que requeriam a administração endovenosa de glicose. A avaliação hormonal revelou deficiência secundária de cortisol e de GH. Os outros hormônios hipofisários eram normais e a tomografia de sela túrcica foi normal. Após o tratamento com glicocorticóide, houve desaparecimento dos episódios hipoglicêmicos (5).

Todos estes casos ilustram episódios de hipoglicemia, associados a hipopituitarismo, que se desenvolveram durante a gestação ou no período pósparto, não havendo relato de instabilidade hemodinâmica em nenhum momento. Em todos, os episódios de hipoglicemia desapareceram após o tratamento com glicocorticóides. Isso demonstrou uma relação de causa e efeito entre hipocortisolismo e hipoglicemia. Em uma das pacientes, o estudo histopatológico da hipófise revelou o diagnóstico de hipofisite linfocítica. Além disso, nas três pacientes, o hipopituitarismo apresentava algumas particularidades, sugestivas de acometimento hipofisário auto-imune. Sabe-se que, nas agressões hipofisárias de natureza múltipla (tumores, radioterapia, isquemia hipofisária), observamos, em geral, uma ordem de disfunção hormonal, sendo o setor produtor de $\mathrm{GH}$ o mais precocemente acometido seguido pelo setor gonadotrófico. Segue-se a isto o comprometimento do setor corticotrófico, tireotrófico e finalmente lactotrófico (6). Essa ordem não foi respeitada tanto nos pacientes acima descritos como naqueles apresentados inicialmente. Isso nos leva a pensar numa agressão celular específica do tipo auto-imune, na qual não se observa, em geral, a ordem clássica de acometimento da função hipofisária.

A hipofisite linfocitária é uma patologia que acomete, preferencialmente, as mulheres com idade média de 34 anos (7). Ocorre, um pouco mais tardiamente, por volta da $5^{\circ}$ década, nos pacientes do sexo masculino. A maioria dos casos está relacionada à gestação, ocorrendo principalmente no $3^{\circ}$ trimestre e no período pós parto, embora existam relatos de casos na pós-menopausa. O quadro clínico é decorrente, princi- 
palmente, da disfunção hormonal e/ou do efeito de massa provocado pelo processo inflamatório hipofisário. $\mathrm{O}$ setor da adenohipófise mais comprometido na hipofisite é o corticotrófico, sendo essa patologia a causa mais comum de deficiência isolada de ACTH (8). Não é incomum o paciente apresentar deficiência de cortisol isolada ou associada a de GH ou TSH e preservação do setor gonadotrófico. $\mathrm{O}$ processo inflamatório pode se estender pela haste até o infundíbulo e causar diabetes insipidus em 14\% a 19\% dos casos. Alguns autores relatam a presença de massa hipofisária em até $95 \%$ dos pacientes com hipofisite e, em decorrência disso, os pacientes podem apresentar cefaléia e alterações do campo visual (9). O "tumor", causado pelo edema e processo inflamatório, pode desaparecer espontaneamente devido à evolução para fibrose que substitui, completa ou parcialmente, as células hipofisárias normais. Nessa fase mais tardia, pode não restar seqüela funcional ou radiológica ou podem coexistir imagem radiológica de sela vazia e hipopituitarismo parcial ou total. Portanto, os achados radiológicos variam desde massa hipofisária, com ou sem espessamento da haste, até sela vazia, podendo ser encontradas, no meio desse espectro, hipófise e haste completamente normais $(7,9)$.

A suspeita diagnóstica de hipofisite deve ser levantada nos pacientes com massa hipofisária e espessamento da haste e infundíbulo (esses dados não são encontrados em processo pituitário tumoral), massa hipofisária isolada, sela vazia ou mesmo hipófise normal associadas a deficiência predominante do setor corticotrófico. Entretanto, o diagnóstico definitivo de hipofisite linfocitária é histopatológico $(6,7)$.

Na patogênese da doença estão envolvidos processos de autoimunidade e, em 20 a 30\% dos casos, existe associação com outras doenças autoimunes, sendo a mais comum a tireoidite de Hashimoto $(7,10-12)$.

Embora não tenhamos, nos dois pacientes apresentados, o diagnóstico histológico de hipofisite linfocitária, acreditamos que ambos sejam exemplos dessa patologia, devido ao comprometimento importante do setor corticotrófico e à ausência de lesão tumoral nos estudos radiológicos. A questão colocada é por que esses pacientes apresentam hipoglicemia enquanto, como já dissemos anteriormente, outros pacientes adultos com hipopituitarismo de outras etiologias não manifestam esse sintoma? Embora não exista essa resposta na literatura, acreditamos que isto seja devido à gravidade do hipocortisolismo, decorrente de uma destruição maciça e preferencial do setor corticotrófico. Poderia haver, também, insuficiência adrenal primária devido a adrenalite auto-imune concomitante. Em um dos pacientes, fizemos o teste de estímulo adrenal com a cortrosina depot e observamos cortisol de 9,6ug/dl. Embora esta seja uma resposta deficitária, esse valor sugere a presença de certa reserva adrenal, e poderíamos, eventualmente, documentar uma resposta normal do cortisol se prolongássemos o estímulo adrenal. De qualquer forma, a gravidade do hipocortisolismo causado pela deficiência importante de ACTH ou pela deficiência conjunta primária e secundária da adrenal é que explicaria a ocorrência da hipoglicemia.

Outro aspecto interessante nesses pacientes, para o qual gostaríamos e chamar a atenção, é que eles apresentavam hipotireoidismo com T4 livre baixo e TSH elevado associado ao hipocortisolismo secundário. Isto poderia ser decorrente da associação de duas doenças autoimunes: tireoidite e hipofisite. Entretanto, em um dos pacientes, o TSH normalizou após a correção do hipocortisolismo. Sabe-se que o cortisol é um modulador fisiológico da liberação do TSH e nos casos de hipocortisolismo, o diagnóstico de hipotireoidismo primário só deve ser feito se o TSH persistir elevado após a correção do hipocortisolismo. Como e de que forma o hipocortisolismo causa esse aumento de TSH? A maioria dos autores acredita que o cortisol exerce uma retroalimentação negativa a nível do hipotálamo, no TRH, e que os pacientes com hipocortisolismo têm uma resposta do TSH ao TRH do tipo hipotalâmica, como a observada nos nossos pacientes (13). Uma outra maneira de explicar a subida do TSH em casos de hipocortisolismo seria imaginar que, como o cortisol é um modulador do sistema autoimune, a deficiência de cortisol poderia levar a muitas doenças imunológicas, inclusive a tiroidite linfocitária. Um dos nossos pacientes era portador de hipotiroidismo primário com anticorpos antitireoideanos negativos, já que a reposição com glicocorticóide não corrigiu o TSH.

O último ponto a ser considerado é a presença de calcificação de pavilhões auriculares nos dois pacientes. Essa anormalidade, descrita inicialmente nos casos de Doença de Addison ou insuficiência adrenal primária, já foi relatada em pacientes com insuficiência adrenal secundária e tem fisiopatologia desconhecida (14).

Concluímos que os dois pacientes são exemplos de hipopituitarismo parcial associado a hipoglicemia de jejum espontânea. Havia, nos dois casos, deficiência dos setores corticotrófico e somatotrotófico, com grande acometimento da produção de cortisol. Em função dessas características, fizemos a suposição de que o comprometimento da função hipofisária foi provocado por um processo autoimune. Os dois pacientes foram medicados com doses substitutivas de glicocorticóide e, 2 anos após o início do tratamento substitutivo, estão se mantendo assintomáticos. 


\section{DISCUSSÃO ABERTA}

Dra. Marcia Nery (Médica Assistente do Serviço de Endocrinologia e Metabologia HCFMUSP): Por que a atividade de renina plasmática está baixa no caso 1 ?

Dra. Catarina Brasil D'Alva Rocha: Também nos chamou a atenção a presença de hipoaldosteronismo hipo-reninêmico em um dos nossos pacientes. A hiponatremia, que pode ocorrer nos pacientes com insuficiência adrenal secundária, pode ser devida à secreção inapropriada de HAD, já que o cortisol também é um modulador fisiológico da secreção do hormônio antidiurético (HAD), ou à presença de hipoaldosteronismo hipo-reninêmico. Os pacientes com insuficiência adrenal secundária têm hipoaldosteronismo com renina baixa e a reposição de glicocorticóide por um período prolongado regulariza a secreção de renina e aldosterona. A idéia é de que o cortisol exerce um efeito permissivo na liberação de renina (15).

Dra. Maria Adelaide Albergaria Pereira: Não existem, na literatura, referências muito esclarecedoras sobre o papel do cortisol na secreção da renina. Alguns artigos demonstram, através de testes de postura e de injeção de angiotensina e de ACTH, que a deficiência de cortisol impede a liberação do sistema renina angiotensina. A reposição de cortisol provoca uma liberação da renina. Gostaria de chamar a atenção para o fato de que essa renina baixa talvez seja mais um dado sugestivo de ausência de comprometimento primário da função adrenal.

Prof. Dr. Wiliam Nicolau (Prof. Associado da Faculdade de Medicina USP): Gostaria de chamar a atenção para o fato de que nos testes de indução de hipoglicemia pela infusão de insulina foram utilizadas doses muito altas de insulina. Isto pode impedir que se evidencie o papel importante que os hormônios contra-reguladores têm na correção da hipoglicemia.

Dra Catarina Brasil D'Alva Rocha: De qualquer forma, estes testes evidenciam que a dissipação da insulina é um fator importante na recuperação da hipoglicemia. Se isto não ocorrer teremos hipoglicemia persistente. Se, nessas condições, houver deficiência dos hormônios contra-reguladores a hipoglicemia será mais severa e persistente.

Prof. Dr. Marcello Delano Bronstein (Professor Livre-Docente do HC-FMUSP): Na revisão que você fez de hipofisite qual é o percentual de pacientes com anticorpos anti-tireoideanos positivos $\mathrm{e}$ anti-adrenal positivo?

Dra. Catarina Brasil D'Alva Rocha: A metodologia para detecção de anticorpos anti-adrenais é mais recente e não existem dados da sua prevalência nos casos de hipofisite. A prevalência de outras doenças autoimunes está em torno de $20 \%$ a $30 \%$ dos casos, sendo a principal delas a tireoidite linfocitária.

Prof. Dr. Marcello Delano Bronstein: O diagnóstico de hipofisite, naturalmente, é presumível, mas eu acho que é, sem dúvida, o primeiro a ser feito nesses pacientes. Você não teve nenhuma resposta ao teste agudo da cortrosina, mesmo usando doses altas de ACTH. Isto poderia ser causado pela ausência do estímulo crônico sobre as adrenais que impediria uma resposta aguda. Entretanto, é possível que haja, além da insuficência de ACTH, uma deficiência primária da adrenal com insuficiência de aldosterona.

Dra. Catarina Brasil D'Alva Rocha: Realizamos, no caso 1 , o teste de Cortrosina depot e observamos cortisol de $9,6 \mu \mathrm{g} / \mathrm{dl}$, após o estímulo. Embora esperássemos resposta maior do cortisol, para níveis superiores a $18 \mu \mathrm{g} / \mathrm{dl}$, este foi o maior nível de cortisol apresentado pelo paciente, que mantinha níveis basais menores do que $1 \mu \mathrm{g} / \mathrm{dl}$. Temos duas possibilidades: 1- Não existe insuficiência adrenal primária concomitante e se prolongarmos o teste vai haver uma reposta normal ou; 2- Existe insuficência adrenal primária parcial. De qualquer forma o paciente é portador de insuficência adrenal secundária.

Dra Maria Adelaide Albergaria Pereira: O hipoaldosteronismo apresentado pelo caso 1 é hiporeninêmico e, portanto, não é sugestivo de insuficiência primária das adrenais. Ele parece ser decorrente, como já discutimos, da insuficiência adrenal secundária.

Prof. Dr. Marcello Delano Bronstein: Com relação ao hipotireoidismo, você disse que o TSH elevado pode ser secundário à deficiência de cortisol. De qualquer forma você poderia ter tireoidite autoimune associada a hipofisite e melhora do processo inflamatório após a administração de corticóide, com normalização do TSH. Acho difícil saber o que está acontecendo nesses casos. Além disso acho que você não pode afastar completamente o hipotireoidismo hipotalâmico, mesmo porque os pacientes apresentam 
uma resposta do tipo hipotalâmica do TSH ao TRH. Como sabemos, pode existir comprometimento infundibular na hipofisite e, portanto, poderíamos ter um hipotireoidismo terciário em decorrência do acometimento do hipotálamo.

Dra. Catarina Brasil D'Alva Rocha: Você consideraria essa hipótese, mesmo havendo normalização do TSH no caso 1, com a introdução do glicorticóide?

Prof. Dr. Marcello Delano Bronstein: Poderia ter ocorrido correção do processo autoimune e melhora do processo inflamatório.

Dra. Maria Adelaide Albergaria Pereira: Gostaria de chamar a atenção para o fato de que usamos doses substitutivas de glicorticóides e não doses farmacológicas, utilizadas quando se tratam as doenças autoimunes. Não acredito que com doses fisiológicas de corticóide possamos reverter, com tanta rapidez, o processo inflamatório. A nossa conclusão foi a de que em um dos pacientes o TSH estava elevado apenas pela deficiência do cortisol e no outro paciente havia, também, uma insuficiência tireoideana primária com anticorpos negativos. A resposta hipotalâmica do TSH ao TRH, como já disse a Dra. Catarina, é observada nos pacientes com hipocortisolismo. É importante chamar a atenção para o fato de que o diagnóstico de hipotireoidismo primário não deve ser realizado nos pacientes com deficiência de cortisol e níveis elevados de TSH, antes de corrigirmos o hipocortisolismo.

Profa. Dra. Berenice Bilharinho Mendonça (Professora Associada da Faculdade de Medicina USP): Acho que podemos afirmar, com bastante segurança, que esses pacientes são portadores de insuficiência adrenal primária. Na nossa experiência todos os pacientes com insuficiência adrenal secundária à síndrome de Sheehan respondem ao teste da Cortrosina aguda. Em nenhum dos 15 a 20 pacientes com esse diagnóstico, nos quais fizemos esse teste, o cortisol permaneceu em $1 \mu \mathrm{g} / \mathrm{dl}$. Quando se utiliza a Cortrosina depot o cortisol chega a valores de $50 \mu \mathrm{g} / \mathrm{dl}$ e não 18 ou $20 \mu \mathrm{g} / \mathrm{dl}$. Entretanto esses pacientes não apresentam clínica ou laboratório de insuficiência adrenal primária, já que têm potássio normal e não apresentam distúrbios hemodinâmicos. Eles poderiam ter um comprometimento primário com preservação do setor produtor de aldosterona. Acho que seria interessante fazer o estudo dos anticorpos antiadrenais nesses dois pacientes.
Dra. Maria Adelaide Albergaria Pereira: Dra. Berenice, você realizou o teste da Cortrosina simples em pacientes com síndrome de Sheehan e com deficiência de ACTH, provavelmente não tão intensa como a dos pacientes com hipofisite. Está bem documentado, na literatura, casos de pacientes com insuficiência adrenal secundária que não respondem ao teste da Cortrosina aguda e que necessitam do estímulo crônico. Essa ausência de resposta do cortisol no teste de estímulo agudo é vista nos pacientes com deficiência grave de ACTH. Os pacientes com síndrome de Sheehan apresentam, provavelmente, deficiência de ACTH menos importante do que aqueles com hipofisite, onde o comprometimento do setor corticotrófico é mais seletivo e talvez mais intenso. Chamo a atenção, novamente, para $o$ fato de que um dos nossos pacientes tinha deficiência de aldosterona e renina baixa, o que não fala a favor de insuficiência adrenal primária. Acho que podemos afirmar, com certeza, que o paciente é portador de insuficiência adrenal secundária, já que tem cortisol muito baixo associado a níveis baixos de ACTH. Como já discutimos anteriormente, ele também pode ser portador de insuficiência adrenal primária.

Gostaria que ficasse claro para todos que hipoglicemia é incomum em pacientes adultos com hipopituitarismo. Apenas em crianças, com deficiência de GH ou de GH e cortisol, ocorre hipoglicemia de jejum. Na revisão feita pela Dra. Catarina ela verificou que episódios hipoglicêmicos são descritos, principalmente, em casos de pacientes adultos com hipopituitarismo associado à hipofisite linfocitária. Será que nesses casos a deficiência de cortisol é mais intensa devido a um comprometimento preferencial do setror corticotrófico?

Infelizmente, não dispunhamos da determinação dos anticorpos antiadrenais, para a detecção de adrenalite autoimune. Talvez possamos fazer, brevemente, a determinação do anticorpo anti-21hidroxilase.

Prof. Dr. Marcello Delano Bronstein: A hipótese que vocês estão colocando em primeiro lugar é que realmente o TSH elevado seja secundário ao hipoadrenalismo. Como está, em geral, a função tireoidiana na doença de Addison?

Dra. Catarina Brasil D'Alva Rocha: Existem muitos pacientes com hipocortisolismo e TSH elevado ou muito elevado e T4-livre baixo ou no limite inferior da normalidade. Essa alteração pode ser secundária ao hipocortisolismo, sem doença primária da tireóide, e é corrigida pela administração de glicorticóide. 
Prof. Dr. Wilian Nicolau: Em situações de hipercortisolismo temos diminuição do TSH e falta de resposta desse hormônio à administração de TRH. No casos de hipocortisolismo nós podemos ter o inverso. Os pacientes com hipocortisolismo poderiam ter um TSH com atividade biológica reduzida.

\section{REFERÊNCIAS}

1. Bolli GB, Fanelli CG. Physiology of glucose counterregulation to hypoglycemia. Endocrinol Metab Clin North Am 1999:28:467-94.

2. De Feo P, Perriello G, De Cosmo S, Ventura MM, Campbell PJ, Brunetti P, et al. Comparison of glucose counterregulation during short term and prolonged hypoglycemia in normal humans. Diabetes 1986;35:563-9.

3. Richtsmeier AJ, Henry RA, Bloodworth MB, Ehrlich EN. Lymphoid hypophysitis wiyh selective adrenocorticotropic hormone deficiency. Arch Intern Med 1980; $140: 1243-5$.

4. Ober KP. Case report: postpartum hypopituitarism with preservation of the pituitary-ovarian axis. Am J Med Sci 1990;299(4):257-9.

5. Smallidge RC, Corrigan DF, Thomason AM, Blue PW. Hypoglicemia in pregnancy: Ocurrence due to adrenocorticotropic hormone and growth hormone deficiency. Arch Intern Med 1980; 140:564-5.

6. Cosman F, Post DK, Holub DA, Wardlaw SL. Lymphocitic hypophyitis. Report of 3 new cases and rewiew of literature. Medicine 1986;68:240-56.

7. Thoudou E, Asa SL, Kontogeorgos G, Kovacs K, Horvath E, Ezzat S. Clinical case seminar: lymphocytic hypophysitis: clinicopathologycal findings. J Clin Endocrinol Metab 1995;80:2302-11.

8. Rock P. Lymphocitic Hypophysitis. Curr Opin Endocrinol Diabetes 1997:4:115-23.
9. Powrie JK, Powell M, Ayers AB, Lowy C, Sönksen PH. Lymphocitic adenohypophysitis: magnetic resonance imaging features of two new cases and a rewiew of the literature. Clin Endocrinol 1995;42:315-22.

10. Osawa Y, Shishiba Y. Recovery from lymphocitic hypophysitis associated with painless thyroiditis: clinical implications of circulanting antipituitary antibodies. Acta Endocrinol (Copenh) 1993; 128:493-8.

11. Barkan AL, Kelch RP, Marshall JC. Isolated gonadotrope failure in the polyglandular autoimmune syndrome. $\mathbf{N}$ Engl J Med 1985;312:1535-40.

12. Kojima I, Nejima I, Ogata E. Isolated adrenocorticotropin deficiency associated with polyglandular failure. J Clin Endocrinol Metab 1982;54:182-6.

13. Shimizu M, Takazakura E, Haykawa H, Kawai K, Muramoto $\mathrm{S}$, Yoshida $\mathrm{K}$, et al. La réacion de la thyrotropine à la thyrotropine-releasing hormone chez les addisoniens. Ann Endocrinol (Paris) 1975;36:317-20.

14. Barkan A, Glantz I. Calcification of auricular cartilages in patients with hypopituitarism. J Clin Endocrinol Metab 1982;55:354-7.

15. Merrian GR, Baer L. Adrenocorticotropin deficiency: Correction of hyponatremia and hypoaldosteronism with chronic glucocorticoid therapy. J Clin Endocrinol Metab 1980;50:10-4.

\section{Endereço para correspondência:}

Maria Adelaide Albergaria Pereira

Av. Dr. Enéas de Carvalho Aguiar, 255

PAMB $8^{\circ}$ andar - Bloco 3

05403-000 São Paulo, SP

Fax: 3088-6964

e.mail:sImnendoc@hcnet.usp.br 